\title{
Trace and Macro Elements Bioaccumulation in the Muscle and Liver Tissues of Alburnus chalcoides from the South Caspian Sea and Potential Human Health Risk Assessment
}

\author{
Masoud Sattari ${ }^{1,2, ~ *, ~ J a v i d ~ I m a n p o u r ~ N a m i n ~}{ }^{1}$, Mehdi Bibak ${ }^{1}$, Mohammad Forouhar Vajargah ${ }^{1}$, \\ Caterina Faggio $^{3}$, Marzieh Soroush Haddad ${ }^{1}$ \\ ${ }^{1}$ Fisheries Department, Faculty of Natural Resources, University of Guilan, Sowmeh Sara, Iran \\ ${ }^{2}$ Department of Marine Sciences, Caspian Sea Basin Research Center, University of Guilan, Rasht, Iran \\ ${ }^{3}$ Department of Chemical, Biological, Pharmaceutical and Environmental Sciences, University of Messina, Messina, Italy
}

Email address:

msattari@guilan.ac.ir (M. Sattari)

${ }^{*}$ Corresponding author

\section{To cite this article:}

Masoud Sattari, Javid Imanpour Namin, Mehdi Bibak, Mohammad Forouhar Vajargah, Caterina Faggio, Marzieh Soroush Haddad. Trace and Macro Elements Bioaccumulation in the Muscle and Liver Tissues of Alburnus chalcoides from the South Caspian Sea and Potential Human Health Risk Assessment. Journal of Energy, Environmental \& Chemical Engineering. Vol. 4, No. 1, 2019, pp. 13-20.

doi: $10.11648 /$ j.jeece.20190401.13

Received: March 21, 2019; Accepted: May 6, 2019; Published: June 13, 2019

\begin{abstract}
Danube bleak Alburnus chalcoides (Güldenstädt, 1772) is a commercially important fish species in the southern part of the Caspian Sea. However, little is known about the concentrations of trace element (TE) in its muscles. To address the issue, we caught $77 \mathrm{~A}$. chalcoides specimens at three different fishing regions (including Astara, Anzali, Kiashahr) of the southern shoreline of the Caspian Sea from September 2017 through June 2018. An inductively coupled plasma optical emission spectrometry (ICP-OES) was used to measure element concentrations in fish muscle and liver tissues. In the case of liver, 27 elements were detected. So that, 9 elements including $\mathrm{Ag}, \mathrm{Ba}, \mathrm{Be}, \mathrm{Ce}, \mathrm{Co}, \mathrm{Sc}, \mathrm{U}, \mathrm{V}$ and $\mathrm{Y}$ which were not detected in liver. In the case of muscle tissue, 22 elements were detected and 14 elements including $\mathrm{Ag}, \mathrm{Ba}, \mathrm{Be}, \mathrm{Bi}, \mathrm{Cd}, \mathrm{Ce}, \mathrm{Co}, \mathrm{La}, \mathrm{Li}, \mathrm{Sc}$, $\mathrm{U}, \mathrm{V}, \mathrm{W}$ and $\mathrm{Y}$. The element values in the muscle of $A$. chalcoides were significantly lower $(\mathrm{p}<0.05)$ than the maximum permitted levels suggested by International standards.
\end{abstract}

Keywords: A. chalcoides, Caspian Sea, Trace Elements, Human Health

\section{Introduction}

Heavy metals are introduced to aquatic environments through natural and anthropological activities. These elements may sink toward the bottom sediment or accumulate directly in marine ecosystem. The accumulation process is affected by different environmental and biological factors such as salinity, temperature, season, size, sex and species. Heavy metals enter aquatics through skin, gill and food. The strict periodic surveillance of these elements is therefore advisable. Toxic metals pollution is less visible and direct than other types of marine pollution but its effects on marine ecosystems and humans are intense and very extensive [1]. Fish are notorious for their ability to concentrate heavy metals in their muscles and they also play important role in human nutrition. So, it is important to carefully screen the bioaccumulation and exposure levels of heavy metal to ensure that unnecessary elevated level of some toxic metals is not being transferred to human through fish consumption. The presence of metal in aquatic environment may lead to its accumulation in organisms through different mechanisms: via the direct uptake from water through gill or skin (bio-concentration), via the uptake of suspended particles (ingestion) and via the consumption of contaminated food (bio-magnification) [2]. More attention has been paid on developed and developing countries about anomalous distribution of metals in the water, sediments and fishes which are important in understanding the behavior of the metals and also the swapping between the sediments and 
the water column [3].

The Caspian Sea is the largest land - enclosed drainage in the world, which supports substantial fisheries. However, it has been endangered by massive loads of contaminants. The various environmental pollutants produced by natural and anthropogenic sources are discharged from coastal catchment $[4,5]$. The hazardous effects of heavy metals on human health are reviewed by international organizations such as the WHO. Bioaccumulation is defined as an increase in the concentration of pollutants by an increase in age, which sometimes is reflected in the size of the organisms [6]. Metal bioaccumulation is influenced by various environmental and biological factors particularly the feeding source $[7,8]$.

Danube bleak Alburnus chalcoides (Güldenstädt, 1772) belonging to Actinopterygii (ray-finned fishes), order Cypriniformes (Carps), family Cyprinidae (Minnows or carps), subfamily Alburninae is a Freshwater, brackish, pelagic and potamodromous fish (Ref. 51243) in temperate regions. $\left(5^{\circ} \mathrm{C}-20^{\circ} \mathrm{C}\right)$ (Ref. 12468). Its name originates from the city of Al Bura, where the fish was known (Ref. 45335). Its distribution: Europe and Asia: Caspian (mostly western to southern coast, rarely found in Ural and Volga). Populations from Aral Sea basin might belong to this species (Ref. 59043). It is reported from the Black Sea basin (Ref. 58342). Max length: $40.0 \mathrm{~cm}$ TL male/unsexed; (Ref. 556); common length: $20.0 \mathrm{~cm} \mathrm{TL}$ male/unsexed; (Ref. 1441); common length: $28 \mathrm{~cm}$ TL (female). It Inhabits lower reaches of rives, coastal lakes, estuaries, and brackish areas of sea and occur close to surface and tolerate salinities up to 14 ppt. Adults predominantly prey on planktonic crustaceans, terrestrial insects and small fish while larvae and young juveniles feed on zooplankton, algae and insect larvae. Spawn in small rivers or streams with heavy current on gravel bottom.

There are numerous reports on heavy metal bioaccumulation in aquatic environments of Iran including
Bibak et al. (2018) [9] who assessed element accumulation in sediments from the northern Persian Gulf; Eslami et al., (2011) [10] heavy metals in muscle and liver of Perca fluviatilis and Tinca tinca in Anzali Wetland; Heydari et al. (2011) [11] on Acipenser stellatus, Monsefrad et al., (2012a, 2012b) [12,13] on Rutilus frisii kutum. There are also some reports on TE concentrations in R. kutum [12-15], but there is no report on TE contamination in Danube bleak, $A$. chalcoides. Therefore, the aim of this study was to determine the levels of some trace elements (TEs) in edible parts of $A$. chalcoides collected from the coast of the Caspian Sea and compare TE levels in fish caught from the different geographical localities as well as to define risk assessment of these elements for human health.

\section{Material and Methods}

This study was conducted at five fisheries areas including Astara: $38^{\circ} 42^{\prime} 25^{\prime \prime} \mathrm{N}, 48^{\circ} 86^{\prime} 87^{\prime \prime} \mathrm{E}$, Anzali: $37^{\circ} 46^{\prime} 39^{\prime \prime} \mathrm{N}$, $49^{\circ} 47^{\prime} 99^{\prime \prime}$ E, Kiashahr: $37^{\circ} 42^{\prime} 20^{\prime \prime}$ N, 49 $94^{\circ}$ 95" E, Sari: $36^{\circ} 78^{\prime} 39^{\prime \prime} \mathrm{N}, 53^{\circ} 03^{\prime} 99^{\prime \prime} \mathrm{E}$ and Torkaman port $36^{\circ} 89^{\prime} 28^{\prime \prime}$ $\mathrm{N}, 54^{\circ} 04^{\prime} 64^{\prime \prime} \mathrm{E}$ along the southern shoreline of the Caspian Sea.

Samplings were carried out from September 2017 to June 2018 and a total of 77 specimens of A. chalcoides were collected. The specimens were transported to the Fish Biology Laboratory, University of Guilan, Sowmeh Sara, Iran using a styrofoam cooler box at $4^{\circ} \mathrm{C}$. Fish were washed using distilled water, dissected and pieces of muscle and liver tissues were placed in an oven at $80^{\circ} \mathrm{C}$ for $18 \mathrm{~h}$ to dry. Age determination was carried out using fish scale during the process (Table 1). To extract elements $0.5 \mathrm{~g}$ of each tissue was digested in $10 \mathrm{ml} 65 \%$ nitric acid using a microwave oven, passed through the Whatman filter paper No. 40 and diluted with distilled water to the required volume.

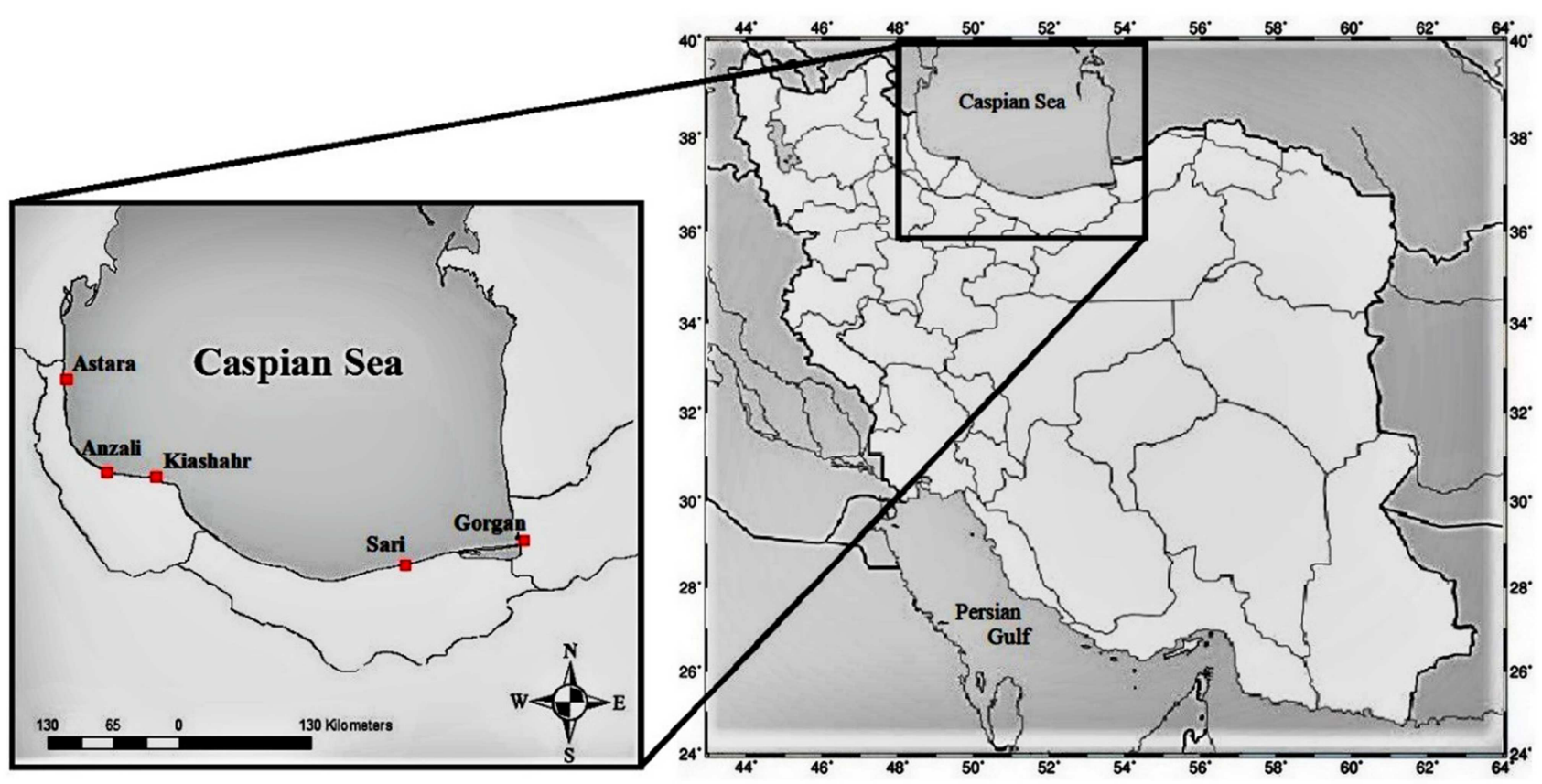

Figure 1. Location of Danube bleak, A. chalcoides sampling areas in the southern coasts of the Caspian Sea. 
An inductively coupled plasma - optical emission spectrometry (ICP-OES) (Zarazma Co. Tehran, Iran) was used for the determination of TE concentrations in the samples. Instrumental detection limits for trace elements was $0.02 \mathrm{mg} \mathrm{kg}^{-1}$, and for major elements (Al, Ca, Fe, K, Mg, $\mathrm{Mn}, \mathrm{Na}$ and $\mathrm{Si}$ ) was $0.1 \mathrm{mg} \mathrm{kg}^{-1}$.

The concentrations of TEs were expressed as the metal selectivity index (MSI) for each tissue.

$$
\mathrm{MSI}=\frac{A}{T} \times 100
$$

$\mathrm{A}=$ absolute concentration of a metal in a tissue

$\mathrm{T}=$ Total concentration of all TEs in that tissue

Statistical analysis
Thirty six trace and major elements were assayed using inductively coupled plasma - optical emission spectrometry (ICP-OES) and then examined statistically excluding Ag, Ba, $\mathrm{Cd}, \mathrm{Co}$ and Mo which were not detected by ICP-OES (Tables 3 \& 4). After examining the normality of the data and homogeneity of variances, One-Way analysis of variances was performed by considering the fish age as a covariate (ANCOVA) to assess possible variability of element levels (Table 3). In cases where homogeneity of variances was not met, the Kruskal-Wallis test was employed (Table 4) [16]. All statistical analyses were performed using SPSS software (SPSS Inc., Chicago, IL). The significant level was considered as $\alpha=0.05$.

Table 1. Morphometric characteristics of Alburnus chalcoides from the study regions of the South Caspian Sea.

\begin{tabular}{|c|c|c|c|c|c|c|c|c|c|}
\hline values & $\begin{array}{l}\text { Total weight } \\
(\mathrm{g})\end{array}$ & $\begin{array}{l}\text { Total length } \\
(\mathrm{cm})\end{array}$ & $\begin{array}{l}\text { Fork length } \\
(\mathrm{cm})\end{array}$ & $\begin{array}{l}\text { Standard } \\
\text { length }(\mathrm{cm})\end{array}$ & $\begin{array}{l}\text { Head Length } \\
(\mathrm{cm})\end{array}$ & $\begin{array}{l}\text { Snout length } \\
(\mathrm{cm})\end{array}$ & $\begin{array}{l}\text { Eye diameter } \\
(\mathrm{cm})\end{array}$ & $\begin{array}{l}\text { Body height } \\
(\mathrm{cm})\end{array}$ & $\begin{array}{l}\text { Body width } \\
(\mathrm{cm})\end{array}$ \\
\hline Average & 76.78 & 19.38 & 4.14 & 3.38 & 7.84 & 2.24 & 1.93 & 8.75 & 4.56 \\
\hline Max & 395 & 34 & 2.35 & 2 & 3.80 & 1.00 & 1.00 & 4.90 & 2.10 \\
\hline Min & 15 & 13 & 1.65 & 1.65 & 3.30 & 0.9 & 0.80 & 3.50 & 1.90 \\
\hline SD & 83.92 & 5.17 & 4 & 3.80 & 0.89 & 0.31 & 0.13 & 0.88 & 0.47 \\
\hline
\end{tabular}

Table 2. Maximum permitted concentration in parts per million (ppm) recommended by Codex Alimentarius Commission (FAO \& WHO, amended in 2018), US Food and Drug Administration (FDA, 2011).

\begin{tabular}{ll}
\hline Elements & $\begin{array}{l}\text { Maximum permitted concentration in } \\
\text { parts per million }(\mathbf{p p m})\end{array}$ \\
\hline Lead & 0.5 \\
Cadmium & 2 \\
Arsenic & 0.1 \\
Chromium & 1 \\
Aluminum & 100 \\
Antimony & 1 \\
Tin & 230 \\
Copper & 10 \\
Manganese & 0.5 \\
Zinc & 100 \\
Selenium & 1 \\
\hline
\end{tabular}

\section{Results and Discussion}

In the present study, a total of $77 \mathrm{~A}$. chalcoides specimens were dissected and their muscle and liver tissues were examined for 36 elements including, silver (Ag), aluminum $(\mathrm{Al})$, arsenic (As), Barium (Ba) beryllium $(\mathrm{Be})$, bismuth $(\mathrm{Bi})$, calcium $(\mathrm{Ca})$, cadmium $(\mathrm{Cd})$, cesium $(\mathrm{Ce})$, cobalt $(\mathrm{Co})$, chromium $(\mathrm{Cr})$, copper $(\mathrm{Cu})$, iron $(\mathrm{Fe})$, potassium $(\mathrm{K})$, lanthanum (La), lithium (Li), magnesium $(\mathrm{Mg})$, manganese (Mn), molybdenum (Mo), sodium (Na), nickel (Ni), phosphorus $(\mathrm{P})$, lead $(\mathrm{Pb})$, rubidium $(\mathrm{Rb})$, sulfur $(\mathrm{S})$, antimony (Sb), scandium (Sc), silicon (Si), tin (Sn), strontium (Sr), thorium (Th), titanium (Ti), uranium (U), vanadium $(\mathrm{V})$, tungsten $(\mathrm{W})$, yttrium $(\mathrm{Y})$ and zinc $(\mathrm{Zn})$.

In the case of liver, 27 elements were detected. So that, 9 elements including $\mathrm{Ag}, \mathrm{Ba}, \mathrm{Be}, \mathrm{Ce}, \mathrm{Co}, \mathrm{Sc}, \mathrm{U}, \mathrm{V}$ and $\mathrm{Y}$ which were not detected in liver, were excluded from statistically analyses. In the case of muscle tissue, 22 elements were detected and 14 elements including $\mathrm{Ag}, \mathrm{Ba}$, $\mathrm{Be}, \mathrm{Bi}, \mathrm{Cd}, \mathrm{Ce}, \mathrm{Co}, \mathrm{La}, \mathrm{Li}, \mathrm{Sc}, \mathrm{U}, \mathrm{V}, \mathrm{W}$ and $\mathrm{Y}$ which were not detected in muscle, excluded from analyses. (Tables 3-4).

According to Table 3, in total, 28 elements were detected from the $A$. chalcoides liver. The detected elements in the liver were measured in the three different regions including Astara, Anzali and Kiashahr but their accumulation (except $\mathrm{Cu}, \mathrm{La}$ and $\mathrm{W}$ ) in these regions were not significantly different (ANOVA and Kruskal - Wallis tests, $\mathrm{p}<0.05$ ). Among the regions, 27 elements were detected in Astara, while 25 elements were found in Anzali and 20 elements in Kiashahr, indicating the higher amounts of pollution in Astara than in Anzali and kiashahr respectively. Noteworthy, Astara is closed to some adjacent littoral states of the Caspian Sea which may play an important role in contaminating the sea by exploring and/or exploiting petroleum, reflecting in detection of elements in aquatic organism's body.

Table 3. Concentrations of trace elements in Alburnus chalcoides liver at three fisheries regions of the South Caspian Sea. P is regarded as being significant if $<0.05$.

\begin{tabular}{lllll}
\hline \multirow{2}{*}{$\begin{array}{l}\text { Elemental variables } \\
(\mathbf{p p m})\end{array}$} & Anzali & Astara & Kiashahr & \multicolumn{2}{c}{ Total } \\
\cline { 2 - 4 } & $\begin{array}{l}\text { Mean } \pm \text { SE } \\
\text { Range }\end{array}$ & $\begin{array}{l}\text { Mean } \pm \text { SE } \\
\text { Range }\end{array}$ & $\begin{array}{l}\text { Mean } \pm \text { SE } \\
\text { Range }\end{array}$ & $\begin{array}{l}\text { Mean } \pm \text { SE } \\
\text { Range }\end{array}$ \\
\hline $\mathrm{Ag}$ & $\mathrm{BDL}$ & $\mathrm{BDL}$ & $\mathrm{BDL}$ & $\mathrm{BDL}$ \\
$\mathrm{Al}$ & $1.73 \pm 1.15$ & $3.85 \pm 3.61$ & $2.96 \pm 1.15$ & $3.25 \pm 2.75$ \\
$\mathrm{As}$ & $0.91-2.55$ & $1.48-11.66$ & $1.79-4.11$ & $0.91-11.66$ \\
& $0.04 \pm 0.02$ & $0.04 \pm 0.007$ & $0.06 \pm 0.02$ & $0.05 \pm 0.01$ \\
& $0.03-0.06$ & $0.03-0.05$ & $0.05-0.09$ & $0.03 *-0.09$ \\
\hline
\end{tabular}


16 Masoud Sattari et al.: Trace and Macro Elements Bioaccumulation in the Muscle and Liver Tissues of Alburnus chalcoides from the South Caspian Sea and Potential Human Health Risk Assessment

\begin{tabular}{|c|c|c|c|c|c|}
\hline \multirow[b]{2}{*}{$\begin{array}{l}\text { Elemental variables } \\
\text { (ppm) }\end{array}$} & Anzali & Astara & Kiashahr & Total & \multirow[b]{2}{*}{ P value } \\
\hline & $\begin{array}{l}\text { Mean } \pm \text { SE } \\
\text { Range }\end{array}$ & $\begin{array}{l}\text { Mean } \pm \text { SE } \\
\text { Range }\end{array}$ & $\begin{array}{l}\text { Mean } \pm \text { SE } \\
\text { Range }\end{array}$ & $\begin{array}{l}\text { Mean } \pm \text { SE } \\
\text { Range }\end{array}$ & \\
\hline \multirow{2}{*}{$\mathrm{Ba}$} & $0.03 \pm 0.02$ & $0.09 \pm 0.13$ & $0.06 \pm 0.03$ & $0.07 \pm 0.09$ & \multirow{2}{*}{$0.72 * *$} \\
\hline & $0.01-0.05$ & $0.01-0.38$ & $0.05-0.12$ & $0.01-0.38$ & \\
\hline $\mathrm{Be}$ & $\mathrm{BDL}$ & $\mathrm{BDL}$ & BDL & BDL & - \\
\hline $\mathrm{Bi}$ & $\mathrm{BDL}$ & $\mathrm{BDL}$ & $\mathrm{BDL}$ & $\mathrm{BDL}$ & - \\
\hline \multirow{2}{*}{$\mathrm{Ca}$} & $77.55 \pm 31.18$ & $71.48 \pm 49.12$ & $53.65 \pm 15.48$ & $66.93 \pm 37.91$ & \multirow{2}{*}{$0.74 *$} \\
\hline & $55.5-99.6$ & $29.8-151$ & $34.8-71.5$ & $29.8-151$ & \\
\hline \multirow{2}{*}{$\mathrm{Cd}$} & $0.03 \pm 0.02$ & $0.04 \pm 0.01$ & $0.05 \pm 0.01$ & $0.04 \pm 0.01$ & \multirow{2}{*}{$0.93 * *$} \\
\hline & $0.01-0.05$ & $0.01-0.06$ & $0.05-0.08$ & $0.01-0.08$ & \\
\hline $\mathrm{Ce}$ & $\mathrm{BDL}$ & $\mathrm{BDL}$ & $\mathrm{BDL}$ & $\mathrm{BDL}$ & - \\
\hline Co & BDL & $\mathrm{BDL}$ & $\mathrm{BDL}$ & $\mathrm{BDL}$ & - \\
\hline \multirow{2}{*}{$\mathrm{Cr}$} & $0.08 \pm 0.04$ & $0.31 \pm 0.07$ & $0.15 \pm 0.07$ & $0.13 \pm 0.07$ & \multirow{2}{*}{$0.53^{*}$} \\
\hline & $0.05-0.11$ & $0.04-0.27$ & $0.09-0.27$ & $0.04-0.27$ & \\
\hline \multirow{2}{*}{$\mathrm{Cu}$} & $0.30 \pm 0.17$ & $0.25 \pm 0.10$ & $0.48 \pm 0.21$ & $0.33 \pm 0.17$ & \multirow{2}{*}{$0.11^{*}$} \\
\hline & $0.18-0.43$ & $0.09-0.41$ & $0.2-0.68$ & $0.09-0.68$ & \\
\hline \multirow{2}{*}{$\mathrm{Fe}$} & $9.12 \pm 3.98$ & $8.85 \pm 6.26$ & $13.90 \pm 8.73$ & $10.45 \pm 6.76$ & \multirow[b]{2}{*}{$0.51^{*}$} \\
\hline & $6.3-11.94$ & $4.61-22.04$ & $6.11-25.75$ & $4.61-25.75$ & \\
\hline \multirow[b]{2}{*}{ K } & $139.4 \pm 38.18$ & $119.87 \pm 57.95$ & $159.95 \pm 60.83$ & $135.2 \pm 55.4$ & \multirow{2}{*}{$0.22 * *$} \\
\hline & $112.4-166.4$ & $58.1-241.2$ & $108.9-239.2$ & $58.1-241.2$ & \\
\hline $\mathrm{La}$ & BDL & $\mathrm{BDL}$ & BDL & $\mathrm{BDL}$ & - \\
\hline $\mathrm{Li}$ & BDL & $0.04 \pm 0.01$ & $\mathrm{BDL}$ & $0.04 \pm 0.01$ & $0.65 * *$ \\
\hline & & $0.01-0.05$ & BDL & $0.01-0.05$ & 0.05 \\
\hline $\mathrm{Mg}$ & $20 \pm 10.46$ & $17.88 \pm 7.84$ & $17.77 \pm 4.69$ & $18.17 \pm 6.78$ & $0.93 *$ \\
\hline Mg & $12.6-27.4$ & $7.48-29$ & $14.1-24.3$ & $7.48-29$ & $0.93^{\prime \prime}$ \\
\hline $\mathrm{Mn}$ & $0.08 \pm 0.05$ & $0.10 \pm 0.09$ & $0.08 \pm 0.03$ & $0.09 \pm 0.07$ & $0.73 *$ \\
\hline $\mathrm{Mn}$ & $0.04-0.12$ & $0.05-0.3$ & $0.05-0.12$ & $0.04-0.3$ & $0.13^{*}$ \\
\hline Mo & $0.03 \pm 0.02$ & $0.03 \pm 0.01$ & BDI & $0.04 \pm 0.01$ & $037 * *$ \\
\hline MIO & $0.01-0.05$ & $0.01-0.05$ & BDL & $0.01-0.05$ & $0.3 / \%$ \\
\hline $\mathrm{Na}$ & $73.4 \pm 35.77$ & $53.64 \pm 22.49$ & $85.97 \pm 49.97$ & $66.63 \pm 34.85$ & $039 *$ \\
\hline $\mathrm{Na}$ & $48.1-98.7$ & $27.9-87$ & $50.2-158.5$ & $27.9-158.5$ & $0.39^{*}$ \\
\hline & $0.03 \pm 0.02$ & $0.05 \pm 0.02$ & $0.05 \pm 0.01$ & $0.05 \pm 0.02$ & \\
\hline $\mathrm{Ni}$ & $0.01-0.05$ & $0.01-0.11$ & $0.05-0.08$ & $0.01-0.11$ & $0.81^{* *}$ \\
\hline & $191.95 \pm 108.2$ & $149.5 \pm 55$ & $0.05 \pm 0.01$ & $172.43 \pm 61.83$ & \\
\hline $\mathrm{P}$ & $115.4-268.5$ & $72.6-223$ & $0.05-0.08$ & $72.6-268.8$ & $0.37^{*}$ \\
\hline & $0.06 \pm 0.01$ & $0.05 \pm 0.01$ & & $0.05 \pm 0.01$ & \\
\hline $\mathrm{Pb}$ & $0.05-0.07$ & $0.05-0.09$ & $\mathrm{BDL}$ & $0.05-0.09$ & $0.40^{* *}$ \\
\hline & $0.27 \pm 0.31$ & $0.33 \pm 0.45$ & $0.24 \pm 0.12$ & $0.29 \pm 0.34$ & \\
\hline $\mathrm{Rb}$ & $0.05-0.5$ & $0.05-1.05$ & $0.14-0.4$ & $0.05-1.05$ & $0.66^{* *}$ \\
\hline & $168.9 \pm 32.38$ & $117.7 \pm 33.09$ & $133.6 \pm 43.40$ & $130.4 \pm 38.08$ & \\
\hline $\mathrm{S}$ & 146-191.8 & $74.3-173.1$ & $88.1-189.5$ & $74.3-191.8$ & $0.25^{*}$ \\
\hline $\mathrm{Sh}$ & $0.04 \pm 0.03$ & $0.07 \pm 0.06$ & $0.14 \pm 0.13$ & $0.09 \pm 0.08$ & $0.84 * *$ \\
\hline Sb & $0.02-0.07$ & $0.02-0.21$ & $0.05-0.33$ & $0.02-0.33$ & $0.84^{\mathrm{N}}$ \\
\hline $\mathrm{Sc}$ & $\mathrm{BDL}$ & $\mathrm{BDL}$ & $\mathrm{BDL}$ & $\mathrm{BDL}$ & - \\
\hline $\mathrm{Si}$ & $0.28 \pm 0.21$ & $1.06 \pm 1.20$ & $0.92 \pm 0.73$ & $0.89 \pm 0.97$ & $057 * *$ \\
\hline S1 & $0.13-0.44$ & $0.08-2.91$ & $0.35-1.94$ & $0.08-2.91$ & 0.51. \\
\hline $\mathrm{Sn}$ & $0.05 \pm 0.007$ & $0.05 \pm 0.01$ & & $0.05 \pm 0.01$ & 0 76** \\
\hline Sn & $0.05-0.06$ & 0.05-0.09 & $\mathrm{BDL}$ & $0.05-0.09$ & $0.76^{* *}$ \\
\hline $\mathrm{Sr}$ & $0.38 \pm 0.27$ & $0.34 \pm 0.25$ & $0.24 \pm 0.11$ & $0.32 \pm 0.21$ & $070 *$ \\
\hline $\mathrm{Sr}$ & $0.19-0.58$ & $0.1-0.86$ & $0.12-0.39$ & $0.1-0.86$ & $0.70^{*}$ \\
\hline & $0.06 \pm 0.01$ & $0.04 \pm 0.007$ & & $0.05 \pm 0.01$ & $031 * *$ \\
\hline Th & $0.05-0.07$ & $0.03-0.05$ & $\mathrm{BDL}$ & 0.05-0.09 & $0.31^{\mathrm{Fa}}$ \\
\hline & $0.05 \pm 0.04$ & $0.11 \pm 0.20$ & & $0.08 \pm 0.14$ & \\
\hline $\mathrm{Ti}$ & $0.02-0.09$ & $0.01-0.58$ & $\mathrm{BDL}$ & $0.01-0.58$ & $0.07 \times 7$ \\
\hline & $0.05 \pm 0.01$ & $0.03 \pm 0.01$ & BDL & $0.04 \pm 0.01$ & \\
\hline U & $0.03-0.05$ & $0.01-0.05$ & $\mathrm{BDL}$ & $0.01-0.05$ & $0.30^{* * *}$ \\
\hline V & $\mathrm{BDL}$ & $\mathrm{BDL}$ & $\mathrm{BDL}$ & 0 & 0 \\
\hline W & $\mathrm{BDL}$ & $\begin{array}{l}0.07 \pm 0.05 \\
0.05-0.2\end{array}$ & $\mathrm{BDL}$ & $\begin{array}{l}0.06 \pm 0.04 \\
0.05-0.2\end{array}$ & $0.65^{* *}$ \\
\hline Y & $\mathrm{BDL}$ & $\mathrm{BDL}$ & $\mathrm{BDL}$ & $\mathrm{BDL}$ & - \\
\hline $7 \mathrm{n}$ & $1.55 \pm 1.01$ & $1.42 \pm 0.40$ & $1.77 \pm 0.52$ & $1.55 \pm 0.51$ & $080 *$ \\
\hline $\mathrm{Zn}$ & $0.83-2.27$ & $0.87-1.93$ & $2.46-1.34$ & $0.83-2.46$ & $0.80^{*}$ \\
\hline
\end{tabular}

*. Tested by One - Way ANOVA, **. Tested by Kruskal-Wallis, BDL: below detectable level

According to Table 4, in total, 24 elements were detected in $A$. chalcoides muscle exhibiting fewer number of elements in the fish muscle than in its liver. The detected elements in the muscle were measured in the three different regions 
including Astara, Anzali and Kiashahr but their concentrations in these regions were not significantly different (One - Way ANOVA and Kruskal - Wallis tests, $\mathrm{p}<0.05$ ). Among the regions, 24 elements were detected in Astara, while 20 elements were found in Anzali and 14 elements in Kiashahr, indicating the lower amounts of pollution in kiashahr than in Anzali and Astara respectively.
Noteworthy, Sefidrud River which provides the main source of tap (plumbing) water in this area, and consequently nearly free from industrial and urban effluents, enters the Caspian Sea adjacent to the kiashahr which may be a reason for lower number of detected elements in the fish caught from this region.

Table 4. Concentrations of trace elements in Alburnus chalcoides muscle at three fisheries regions of the South Caspian Sea. P is regarded as being significant if $<0.05$.

\begin{tabular}{|c|c|c|c|c|c|}
\hline \multirow[b]{2}{*}{$\begin{array}{l}\text { Elemental variables } \\
\text { (ppm) }\end{array}$} & Anzali & Astara & Kiashahr & Total & \multirow[b]{2}{*}{ P value } \\
\hline & $\begin{array}{l}\text { Mean } \pm \text { SE } \\
\text { Range }\end{array}$ & $\begin{array}{l}\text { Mean } \pm \text { SE } \\
\text { Range }\end{array}$ & $\begin{array}{l}\text { Mean } \pm \text { SE } \\
\text { Range }\end{array}$ & $\begin{array}{l}\text { Mean } \pm \text { SE } \\
\text { Range }\end{array}$ & \\
\hline $\mathrm{Ag}$ & $\mathrm{BDL}$ & $\mathrm{BDL}$ & $\mathrm{BDL}$ & $\mathrm{BDL}$ & - \\
\hline $\mathrm{Al}$ & $\begin{array}{l}1.44 \pm 1.44 \\
0.42-2.46\end{array}$ & $\begin{array}{l}1.82 \pm 1.40 \\
0.47-3.71\end{array}$ & 3.12 & $\begin{array}{l}1.88 \pm 1.31 \\
0.42-3.71\end{array}$ & $0.63 *$ \\
\hline As & $\mathrm{BDL}$ & $\begin{array}{l}0.06 \pm 0.03 \\
0.05-0.13\end{array}$ & $\mathrm{BDL}$ & $\begin{array}{l}0.06 \pm 0.02 \\
0.05-0.13\end{array}$ & $0.56^{*}$ \\
\hline $\mathrm{Ba}$ & BDL & BDL & BDL & BDL & - \\
\hline $\mathrm{Be}$ & BDL & BDL & BDL & BDL & - \\
\hline $\mathrm{Bi}$ & BDL & $\mathrm{BDL}$ & BDL & BDL & - \\
\hline $\mathrm{Ca}$ & $\begin{array}{l}160.45 \pm 95.24 \\
93.1-227.8\end{array}$ & $\begin{array}{l}158.08 \pm 104.4 \\
72.5-347\end{array}$ & 86.7 & $\begin{array}{l}0.07 \pm 0.05 \\
0.05-0.2\end{array}$ & $0.97 *$ \\
\hline $\mathrm{Cd}$ & $\mathrm{BDL}$ & $\mathrm{BDL}$ & BDL & BDL & - \\
\hline $\mathrm{Ce}$ & $\mathrm{BDL}$ & BDL & $\mathrm{BDL}$ & $\mathrm{BDL}$ & - \\
\hline Co & $\mathrm{BDL}$ & $\mathrm{BDL}$ & $\mathrm{BDL}$ & $\mathrm{BDL}$ & - \\
\hline $\mathrm{Cr}$ & $\begin{array}{l}0.06 \pm 0.02 \\
0.04-0.08\end{array}$ & $\begin{array}{l}0.09 \pm 0.05 \\
0.05-0.19\end{array}$ & 0.14 & $\begin{array}{l}0.09 \pm 0.04 \\
0.04-0.19\end{array}$ & $0.44 *$ \\
\hline $\mathrm{Cu}$ & $\begin{array}{l}0.06 \pm 0.03 \\
0.05-0.13\end{array}$ & $\begin{array}{l}0.05 \pm 0.01 \\
0.03-0.06\end{array}$ & 0.06 & $\begin{array}{l}0.05 \pm 0.01 \\
0.02-0.08\end{array}$ & $0.80 *$ \\
\hline $\mathrm{Fe}$ & $\begin{array}{l}1.32 \pm 1.04 \\
0.58-2.06\end{array}$ & $\begin{array}{l}1.31 \pm 0.46 \\
0.73-2.1\end{array}$ & 1.45 & $\begin{array}{l}1.33 \pm 0.52 \\
0.58-2.1\end{array}$ & $0.97 *$ \\
\hline K & $\begin{array}{l}200.3 \pm 110.5 \\
122.1-278.5\end{array}$ & $\begin{array}{l}277.3 \pm 92.67 \\
180.3-450.6\end{array}$ & 181.6 & $\begin{array}{l}249.6 \pm 93.06 \\
122.1-450.6\end{array}$ & $0.50 *$ \\
\hline $\mathrm{La}$ & $\mathrm{BDL}$ & BDL & BDL & $\mathrm{BDL}$ & - \\
\hline $\mathrm{Li}$ & BDL & BDL & $\mathrm{BDL}$ & BDL & - \\
\hline $\mathrm{Mg}$ & $\begin{array}{l}23.25 \pm 12.65 \\
14.3-32.2\end{array}$ & $\begin{array}{l}26.65 \pm 9.88 \\
17.4-46\end{array}$ & 17.2 & $\begin{array}{l}24.84 \pm 9.56 \\
14.3-46\end{array}$ & $0.69 *$ \\
\hline $\mathrm{Mn}$ & $\begin{array}{l}0.03 \pm 0.02 \\
0.02-0.05\end{array}$ & $\begin{array}{l}0.04 \pm 0.008 \\
0.03-0.05\end{array}$ & BDL & $\begin{array}{l}0.04 \pm 0.01 \\
0.02-0.05\end{array}$ & $0.15^{* *}$ \\
\hline Mo & $\begin{array}{l}0.05 \pm 0.01 \\
0.02-0.08\end{array}$ & $\begin{array}{l}0.05 \pm 0.01 \\
0.02-0.08\end{array}$ & BDL & $\begin{array}{l}0.03 \pm 0.02 \\
0.01-0.05\end{array}$ & $0.71 * *$ \\
\hline $\mathrm{Na}$ & $\begin{array}{l}56.05 \pm 37.26 \\
29.7-82.4\end{array}$ & $\begin{array}{l}58.83 \pm 17.99 \\
37.9-85.3\end{array}$ & 32.2 & $\begin{array}{l}55.25 \pm 21.26 \\
29.7-85.3\end{array}$ & $0.57^{*}$ \\
\hline $\mathrm{Ni}$ & BDL & $\begin{array}{l}0.03 \pm 0.02 \\
0.01-0.06\end{array}$ & $\mathrm{BDL}$ & $\begin{array}{l}0.04 \pm 0.01 \\
0.01-0.06\end{array}$ & $0.25^{* *}$ \\
\hline $\mathrm{P}$ & $\begin{array}{l}236.75 \pm 97.93 \\
167.5-306\end{array}$ & $\begin{array}{l}248.9 \pm 89.3 \\
187.7-426.2\end{array}$ & 141.5 & $\begin{array}{l}234.3 \pm 86.1 \\
141.5-426.2\end{array}$ & $0.57 *$ \\
\hline $\mathrm{Pb}$ & $\begin{array}{l}0.04 \pm 0.01 \\
0.03-0.05\end{array}$ & $\begin{array}{l}0.04 \pm 0.004 \\
0.04-0.05\end{array}$ & BDL & $\begin{array}{l}0.04 \pm 0.007 \\
0.03-0.05\end{array}$ & $0.61 * *$ \\
\hline $\mathrm{Rb}$ & $\begin{array}{l}0.34 \pm 0.41 \\
0.05-0.63\end{array}$ & $\begin{array}{l}0.31 \pm 0.30 \\
0.05-0.73\end{array}$ & BDL & $\begin{array}{l}0.29 \pm 0.29 \\
0.05-0.73\end{array}$ & $0.36^{* *}$ \\
\hline S & $\begin{array}{l}154.3 \pm 61.23 \\
111-197.6\end{array}$ & $\begin{array}{l}163.8 \pm 73.89 \\
105.5-308.8\end{array}$ & 116.8 & $\begin{array}{l}156.5 \pm 64.1 \\
105.5-308.8\end{array}$ & $0.92 * *$ \\
\hline $\mathrm{Sb}$ & $\begin{array}{l}0.03 \pm 0.03 \\
0.01-0.06\end{array}$ & $\begin{array}{l}0.04 \pm 0.04 \\
0.01-0.12\end{array}$ & 0.11 & $\begin{array}{l}0.05 \pm 0.04 \\
0.01-0.12\end{array}$ & $0.38 *$ \\
\hline $\mathrm{Sc}$ & $\mathrm{BDL}$ & $\mathrm{BDL}$ & BDL & BDL & 0 \\
\hline $\mathrm{Si}$ & $\begin{array}{l}0.44 \pm 0.16 \\
0.33-0.56\end{array}$ & $\begin{array}{l}0.43 \pm 0.31 \\
0.12-0.94\end{array}$ & 0.54 & $\begin{array}{l}0.44 \pm 0.26 \\
0.12-0.94\end{array}$ & $0.94 *$ \\
\hline $\mathrm{Sn}$ & $\begin{array}{l}0.07 \pm 0.02 \\
0.05-0.09\end{array}$ & $\begin{array}{l}0.04 \pm 0.01 \\
0.03-0.06\end{array}$ & BDL & $\begin{array}{l}0.05 \pm 0.01 \\
0.03-0.09\end{array}$ & $0.61 * *$ \\
\hline $\mathrm{Sr}$ & $\begin{array}{l}1.36 \pm 1.29 \\
0.45-2.28\end{array}$ & $\begin{array}{l}1.32 \pm 1.09 \\
0.44-3.16\end{array}$ & 0.7 & $\begin{array}{l}1.26 \pm 1.01 \\
0.44-3.16\end{array}$ & $0.87^{*}$ \\
\hline Th & $\mathrm{BDL}$ & $\begin{array}{l}0.05 \pm 0.01 \\
0.03-0.06\end{array}$ & BDL & $\begin{array}{l}0.05 \pm 0.008 \\
0.03-0.06\end{array}$ & $0.68^{* *}$ \\
\hline $\mathrm{Ti}$ & $\begin{array}{l}0.03 \pm 0.02 \\
0.01-0.05\end{array}$ & $\begin{array}{l}0.03 \pm 0.02 \\
0.01-0.05\end{array}$ & BDL & $\begin{array}{l}0.03 \pm 0.02 \\
0.01-0.05\end{array}$ & $0.67 * *$ \\
\hline
\end{tabular}




\begin{tabular}{|c|c|c|c|c|c|}
\hline \multirow[b]{2}{*}{$\begin{array}{l}\text { Elemental variables } \\
\text { (ppm) }\end{array}$} & Anzali & Astara & Kiashahr & Total & \multirow[b]{2}{*}{$P$ value } \\
\hline & $\begin{array}{l}\text { Mean } \pm \text { SE } \\
\text { Range }\end{array}$ & $\begin{array}{l}\text { Mean } \pm \text { SE } \\
\text { Range }\end{array}$ & $\begin{array}{l}\text { Mean } \pm \text { SE } \\
\text { Range }\end{array}$ & $\begin{array}{l}\text { Mean } \pm \text { SE } \\
\text { Range }\end{array}$ & \\
\hline $\mathrm{U}$ & $\mathrm{BDL}$ & $\begin{array}{l}0.05 \pm 0.008 \\
0.05-0.07\end{array}$ & $\mathrm{BDL}$ & $\begin{array}{l}0.05 \pm 0.007 \\
0.05-0.07\end{array}$ & $0.78 * *$ \\
\hline V & BDL & BDL & BDL & $\mathrm{BDL}$ & - \\
\hline W & BDL & BDL & BDL & BDL & - \\
\hline Y & $\mathrm{BDL}$ & $\mathrm{BDL}$ & BDL & BDL & - \\
\hline $\mathrm{Zn}$ & $\begin{array}{l}1.21 \pm 1.12 \\
0.42-2.01\end{array}$ & $\begin{array}{l}1.11 \pm 0.33 \\
0.77-1.49\end{array}$ & 1.89 & $\begin{array}{l}1.22 \pm 0.54 \\
0.42-2.01\end{array}$ & $0.47^{*}$ \\
\hline
\end{tabular}

*. Tested by ANOVA, **. Tested by Kruskal-Wallis, BDL: below detectable level

Table 5. Comparison of trace element concentrations in the muscle of Alburnus chalcoides in this study with those of different fish species from the literatures.

\begin{tabular}{|c|c|c|c|c|c|c|c|c|}
\hline Element & As & $\mathrm{Cu}$ & $\mathbf{P b}$ & Mn & $\mathbf{N i}$ & $\mathbf{F e}$ & $\mathbf{Z n}$ & Reference \\
\hline Fish species & Mean \pm SD & Mean \pm SD & Mean \pm SD & Mean \pm SD & Mean \pm SD & Mean \pm SD & Mean \pm SD & Reference \\
\hline Rutilus caspius & $0.25 \pm 0.08$ & $0.69 \pm 0.40$ & & & & $5.38 \pm 2.29$ & $7.15 \pm 1.5$ & [17] \\
\hline Neogobius gorlap & $0.24 \pm 0.09$ & $2.37 \pm 1.01$ & & & & $7.88 \pm 2.64$ & $10.21 \pm 2.25$ & [17] \\
\hline Esox lucius & & $0.21 \pm 0.02$ & $0.004 \pm 0.0001$ & & & & $2.55 \pm 0.18$ & [18] \\
\hline Alosa caspia & & & $0.34 \pm 0.09$ & & & $1118 \pm 128.9$ & & [19] \\
\hline Alburnus chalcoides & & $1.46 \pm 1.97$ & & $3.2 \pm 4.1$ & 0.42 & $80.9 \pm 66.5$ & $38.5 \pm 30.4$ & [20] \\
\hline R. kutum & $0.04-0.20$ & $1.0-2.72$ & $0.0-0.02$ & $018-1.27$ & $0.02-0.04$ & $7.85-24.72$ & $1.74-3.86$ & [21] \\
\hline A. braschnikowy & $0.06 \pm 0.02$ & $0.01 \pm 0.0$ & $0.03 \pm 0.01$ & $0.06 \pm 0.02$ & $0.01 \pm 0.007$ & $1.63 \pm 1.01$ & $0.75 \pm 0.64$ & [21] \\
\hline A. chalcoides & & & & & & & & Present study \\
\hline Permitted by FAO/WHO & 0.1 & 10 & 0.5 & 0.5 & & & 100 & [22] \\
\hline
\end{tabular}

The amount order of elements in the fish muscle in the present study was $\mathrm{Zn}>\mathrm{Cu}>\mathrm{Pb}>\mathrm{Cd}$ which is different from those reported by Khanipour et al. (2018) [23] in catfish, $(\mathrm{Zn}>\mathrm{Cu}>\mathrm{Cd}>\mathrm{Pb})$ from Anzali Wetland, Southwest Caspian Sea.

Ali et al (2011) [24] studied five elements including $\mathrm{Cr}$, $\mathrm{Cd}, \mathrm{Pb}, \mathrm{Zn}, \mathrm{Cu}$ in the muscle tissues of $A$. chalcoides $(0.52$, $0.1,1.28,11.43$ and $3.38 \mathrm{mg} / \mathrm{kg}$ respectively) which were higher than those reported in the present study $(0.47,0.25$, $0.23,6.1$ and $0.255 \mathrm{mg} / \mathrm{kg}$ respectively).

In the present study, $\mathrm{Ca}$ and $\mathrm{Mg}$ were higher in Astara than in the other regions, while $\mathrm{Cr}, \mathrm{Zn}$, and $\mathrm{Cu}$ had higher levels in kiashahr and $\mathrm{S}, \mathrm{Pb}$ and $\mathrm{K}$ displayed higher concentrations in Anzali. Meanwhile, some elements including Ag, Be, Bi, $\mathrm{Ce}, \mathrm{La}, \mathrm{Sc}, \mathrm{U}, \mathrm{V}$ and $\mathrm{Y}$ were below detectable levels in all the three regions. Furthermore, Li and $\mathrm{pb}$ were not detected in Kiashahr.

Canli and Atli (2003) [25] reported that in some fish species including ..... Pb in muscle tissue was higher than $\mathrm{Cd}$, while in the present study $\mathrm{Cd}$ was higher than $\mathrm{Pb}$ which may be due to different pollution sources.

Arsenic (AS): According to ATSDR (1998a) [26] and EPA (2010) [27] lethal oral doses of As in animals are higher than those in humans. The oral $\mathrm{LD}_{50}$ values for arsenic ranged 15 $112 \mathrm{mg} \mathrm{kg}^{-1}$. The arsenic levels in different fish species were reported by some authors (Table 5). The As mean level ( \pm $\mathrm{SD}$ ) in the present study was $0.06 \pm 0.02 \mathrm{mg} \mathrm{kg}^{-1} \mathrm{~d}$.w. in both muscle and liver tissues of $A$. chalcoides which was lower than those permitted by Codex Alimentarius Commission (Table 2) [22].

Lead $(\mathrm{Pb})$ : Recommended a provisional tolerable weekly intake of $\mathrm{Pb}$ is $0.025 \mathrm{mg} / \mathrm{kg}$ body weight [28], while the maximum $\mathrm{Pb}$ levels in seafood recommended by the European Community is $0.2 \mathrm{mg} \mathrm{kg}^{-1}$ f.w. in fish. $\mathrm{Pb}$ levels in the fish tissues have been documented by some authors
(Table 5). The $\mathrm{Pb}$ levels $( \pm \mathrm{SD}$ ) in the present study were $0.03 \pm 0.01$ and $0.07 \pm 0.01 \mathrm{mg} \mathrm{kg}^{-1}$ in the muscle and liver of $A$. chalcoides respectively which was lower than those permitted by international standards (Table 2) [22].

Copper $(\mathrm{Cu})$ : Recommended PTWI for copper is 3.5 $\mathrm{mg} / \mathrm{kg}$ body weight per week [28]. The mean levels of $\mathrm{Cu}$ in some fish tissues were reported by some authors (Table 5). The mean levels $( \pm \mathrm{SD})$ of $\mathrm{Cu}$ in the present study were 0.06 \pm 0.05 and $0.12 \pm 0.03 \mathrm{mg} \mathrm{kg}^{-1}$ in the muscle and liver of $A$. chalcoides respectively which were lower than those permitted by international standards (Table 2) [22].

Chromium $(\mathrm{Cr})$ : The proposed daily feeding of $\mathrm{Cr}$ is 50 $200 \mu \mathrm{g}$ [29]. The level of $\mathrm{Cr}$ in diet is important in insulin function and also in metabolism of lipids [30]. There are some reports about the mean $\mathrm{Cr}$ levels in different fish species (Table 5). The mean Cr levels $( \pm \mathrm{SD}$ ) in the present study were $0.05 \pm 0.03$ and $0.05 \pm 0.02 \mathrm{mg} \mathrm{kg}^{-1}$ in muscle and liver tissues of $A$. chalcoides respectively which were lower than those permitted by international standards including Codex Alimentarius Commission [22].

Cobalt (Co): There are some reports about the mean Co accumulation in different fish species (Table 5), but In the present study, the mean level ( \pm SD) of cobalt was $0.01 \pm 0.0$ $\mathrm{mg} \mathrm{kg}^{-1}$ in both muscle and liver tissues of $A$. chalcoides.

Iron (Fe): The US National Academy of Science [29]. Proposed the allowance for iron in diet to be $10 \mathrm{mg} / \mathrm{day}$ for elderly persons. The mean concentrations of $\mathrm{Fe}$ in the different species tissues were reported by some authors (Table 5). The mean levels $( \pm \mathrm{SD})$ of $\mathrm{Fe}$ in the present study were $1.63 \pm 1.01$ and $5.68 \pm 2.51 \mathrm{mg} \mathrm{kg}^{-1}$ in the muscle and liver tissues of $A$. chalcoides respectively.

Manganese $(\mathrm{Mn})$ deficiency leads to severe birth defects, convulsions and asthma [31]. There are some reports about the Mn levels in different fish tissues (Table 5). The mean accumulation $( \pm \mathrm{SD})$ of $\mathrm{Mn}$ in the present study was $0.06 \pm$ 
$0.02 \mathrm{mg} \mathrm{kg}^{-1}$ in both muscle and liver of $A$. chalcoides respectively which was lower than those permitted by international standards including Codex Alimentarius Commission [22].

Nickle (Ni): The World Health Organization [32] proposed 100-300 $\mu \mathrm{g} \mathrm{Ni}$ for daily dietary consumption. The maximum $\mathrm{Ni}$ accumulation permitted for fish is $0.5 \mathrm{mg} \mathrm{kg}^{-1}$ based on Bulgarian Food Codex [32]. There are some reports about the tissue Ni levels in some fish species (Table 5). The mean Ni levels $( \pm \mathrm{SD})$ in the present study were measured as $0.01 \pm$ 0.007 and $0.01 \pm 0.0 \mathrm{mg} \mathrm{kg}^{-1}$ in muscle and liver of $A$. chalcoides respectively which were lower than those permitted by international standards such as Codex Alimentarius Commission [22].

Vanadium (V). There is only one report concerning to the vanadium levels $( \pm$ SD) in the fish tissues in Iran [33]. However, in the present study, vanadium was not detected in A. chalcoides muscles, but just found in the fish liver and its mean level $( \pm \mathrm{SD})$ was $0.1 \pm 0.0$.

Zinc ( $\mathrm{Zn}$ ) [29] proposed the PTWI for $\mathrm{Zn}$ to be $7 \mathrm{mg} \mathrm{kg}^{-1}$ body weight/week. The mean concentrations of $\mathrm{Zn}$ in some fish species were reported by some authors from Iran (Table $5)$. The mean zinc levels $( \pm \mathrm{SD})$ in the present study were $0.75 \pm 0.64$ and $1.27 \pm 0.41 \mathrm{mg} \mathrm{kg}^{-1}$ in muscle and liver of $A$. chalcoides respectively which were lower than those recommended by Codex Alimentarius Commission (Table 2) [22].

\section{Conclusion}

In the present study, $\mathrm{Fe}$ exhibited the highest amount in kidney of $A$. chalcoides than the other elements, while $\mathrm{Sr}$ was higher in fish muscle than in liver. It was also found that $\mathrm{pb}$, $\mathrm{Cr}$ and $\mathrm{Cu}$ was lower than levels permitted by international standards [22]. $\mathrm{Pb}$ in fish caught from Kiashahr was lower than the other regions, while $\mathrm{Fe}$ in this region was higher than in the other regions.

\section{References}

[1] Chi, QQ, Zhu GW, Alan L. 2007-Bioaccumulation of heavy metals in fishes from Taihu Lake, China-J. Environ. Sci. 19: $1500-1504$.

[2] Ikem, A, Egiebor, NO 2008, Assessment of trace elements in canned fishes (mackerel, tuna, salmon, sardines and herrings) marketed in Georgia and Alabama (United States of America). Journal of Food Compositional and Analysis. 18, 771-787.

[3] Yang, G. G., Qin, L., Hong, H. H., Liang, g. W., Jia, J. N., Fei, Y. D. 2017. Heavy metals in fish tissues/stomach contents in four marine wild commercially valuable fish species from the western continental shelf of South China Sea. Marine Pollution Bulletin, Marine Pollution Bulletin, 14 (2), 1125-1129.

[4] Yaghobzadeh, Y., Hossein-Nezhad, M., Asadi-Shiran, G., Pourali, M. 2014. An investigation of lead concentration in Rutilus frisii kutum form Caspian Sea; case study of Bandar Anzali and Roodsar, Iran. Journal of Mazandaran University of Medical Sciences, 23 (110): 102-108 [In Persian].
[5] United Nations Environment Programme (UNEP). 2006. Stolberg, F., Borysova, O., Mitrofanov, I., Barannik, V. Eghtesadi, P. Caspian Sea, GIWA Regional assessment 23. University of Kalmar, Kalmar, Sweden, 92p.

[6] Fernandes, D., Bebianno, M. J., Porte, C. 2008. Hepatic levels of metal and metallothioneins in two commercial fish species of the Northern Iberian shelf. Science of the total environment, 391, 159-167.

[7] Agusa, T., Kunito, T., Tanabe, S., Puorkazemi, M., G. Aubrey, D. 2004. Concentrations of trace elements in muscles of sturgeons in the Caspian Sea. Marine Pollution Bulletin, 49, 789-800.

[8] Agusa, T., Kunito, T., Sudaryanto, A., Monirith, I., KanAtireklap, S., Iwata, H., Ismail, A., Sanguansin, J., Muchtar, M., Tana, T. S., Tanabe, S. 2007. Exposure assessment for trace elements from consumption of marine fish in Southeast Asia. Environmental Pollution, 145, 766-777.

[9] Bibak, M., Sattari, M., Agharokh, A., Tahmasebi, S. and Namin, J. I., 2018. Assessing some heavy metals pollutions in sediments of the northern Persian Gulf (Bushehr province). Environmental Health Engineering and Management Journal.

[10] Eslami, S., Moghaddam, A. H., Jafari, N., Nabavi, S. F., Nabavi, S. M. and Ebrahimzadeh, M. A., 2011. Trace element level in different tissues of Rutilus frisii kutum collected from Tajan River, Iran. Biological Trace Element Research, 143, 965-973.

[11] RDA, Recommended dietary allowance (10th ed.) 1989, Washington, DC: National Academic Press.

[12] S. Heydari, S, Imanpour Namin, J, Mohammadi, M and Rad, FM, 2011, Cadmium and lead concentrations in muscles and livers of stellate sturgeon (Acipenser stellatus) from several sampling stations in the southern Caspian Sea, Journal of Applied Ichthyology, 27: 520-523.

[13] Monsefrad, F, Imanpour Namin, J, Heidary, S, 2012, Concentration of heavy and toxic metals $\mathrm{Cu}, \mathrm{Zn}, \mathrm{Cd}, \mathrm{Pb}$ and $\mathrm{Hg}$ in liver and muscles of Rutilus frisii kutum during spawning season with respect to growth parameters. Iranian Journal of Fisheries Sciences, 11 (4): 825-839.

[14] Monsefrad, F., Imanpour Namin, J. and Heidary, S., 2012. Concentration of heavy and toxic metals $\mathrm{Cu}, \mathrm{Zn}, \mathrm{Cd}, \mathrm{Pb}$ and $\mathrm{Hg}$ in liver and muscles of Rutilus frisii kutum during spawning season with respect to growth parameters. Iranian Journal of Fisheries Sciences, 11, 825-839.

[15] Yalsuyi, A. M. and Vajargah, M. F., 2017. Acute toxicity of silver nanoparticles in Roach (Rutilus rutilus) and Goldfish (Carassius auratus). Journal of Environmental Treatment Techniques, 5 (1), pp. 1-4.

[16] Vajargah, M. F., Yalsuyi, A. M., Sattari, M. and Hedayati, A., 2018a. Acute toxicity effect of glyphosate on survival rate of common carp, Cyprinus carpio. Environmental Health Engineering and Management Journal.

[17] Zar, JH 1996, Biostatistical Analysis. 3rd Edition, Prentice Hall, Inc., Upper Saddle River.

[18] Alipour, H., Pourkhabbaz, A. Hassanpour, M. 2016, Determination of metals ( $\mathrm{As}, \mathrm{Cu}, \mathrm{Fe}$, and $\mathrm{Zn}$ ) in two fish species from the Miankaleh wetland. Arch. Pol. Fish. 23: 99105, DOI 10.1515/aopf-2016-0011. 
[19] Imanpour Namin, J, Mohammadi, M, Heydari, Monsef Rad, SF, 2011, Heavy metals $\mathrm{Cu}, \mathrm{Zn}, \mathrm{Cd}$ and $\mathrm{Pb}$ in tissue, liver of Esox lucius and sediment from the Anzali International Lagoon. J. Caspian J. Environ. Sci., Vol. 9 No. 1 pp. 1-8.

[20] Alipour H, Banagar, Gh. R., 2018, Health risk assessment of selected heavy metals in some edible fishes from Gorgan Bay, Iran. Iranian Journal of Fisheries Sciences 17 (1) 21-34.

[21] Mirzajani, AR, Hamidian AH, Karami, M 2016, Metal bioaccumulation in representative organisms from different trophic levels of the Caspian Sea, Iranian Fisheries Research Organization, 15 (3): 1027-1043.

[22] Sattari, M., Namin, J. I., Vajargah, M. F., Bibak, M., Bakhshalizadeh, S., Hedayati, A., Faggio, C. (2018). Trace element concentrations in liver and kidney tissues of the Caspian kutum (Rutilus kutum Kamensky 1901) from South Caspian Sea. International Journal of Aquatic Biology (In press).

[23] FAO/WHO 2018, General standard for contaminants and toxins in food and feed CXS 193-1995, amended in 2018, 65p.

[24] Khanipour, AA, Ahmadi, M, Seifzadeh, M 2018, Study on bioaccumulation of heavy metals (cadmium, nickel, zinc and lead) in the muscle of wels catfish (Silurus glanis) in the Anzali Wetland, Iranian Journal of Fisheries Sciences, 17 (1): 244-250.

[25] Ali. A. A. Elazein., E. M. Alian., M. A. (2011). Investigation of heavy metals pollution in water, sediment and fish at Red Sea- Jeddah Coast- KSA at two different locations. J Appl Environ Biol Sci, 1 (12), pp. 630-637.

[26] Canli, M., Atli, G. 2003. The relationships between heavy metal $(\mathrm{Cd}, \mathrm{Cr}, \mathrm{Cu}, \mathrm{Fe}, \mathrm{Pb}, \mathrm{Zn})$ levels and the size of six Mediterranean fish species. Environmental Pollution, 121, 129-136.
[27] Agency for Toxic Substannces and Disease Registry (ATSDR) 1999. Toxicological profile for mercury. Atlanta, GA: U.S. Department of Health and Human Services, public health service, 1999.

[28] EPA (United States Environmental Protection Agency), 2010, Guidance for Assessing Chemical Contaminant Data for Use in Fish Advisories, Vol. 2, 383p.

[29] FAO/WHO, 2004, "Summary of Evaluations Performed by the Joint FAO/WHO Expert Committee on Food Additives (JECFA 1956-2003)", ILSI Press International Life Sciences Institute.

[30] ZeljkaVisnjic-Jeftic, Ivan Jaric, Ljubinko Jovanovic, Stefan Skoric, Marija Smederevac-Lalic, Miroslav Nikcevic, Mirjana Lenhardt, 2010. Heavy metal and trace element accumulation in muscle, liver and gills of the Pontic shad (Alosa immaculata Bennet 1835) from the Danube River (Serbia). Microchemical Journal, Volume 95, Issue 2, Pages 341-344.

[31] Bratakos, MS, Lazos, ES, Bratakos, SM, 2002, Chromium content of selected Greek foods. The Science of the Total Environment. 290, 47-58.

[32] Stancheva, M, Makedonski, L, Peycheva, K 2014, Determination of heavy metal concentrations of most consumed fish species from Bulgarian Black Sea coast, Bulgarian Chemical Communications, 46 (1): 195-203.

[33] WHO 1992, Cadmium. Environmental Health Criteria, Volume 134 (Geneva: WHO), 280p.

[34] Janadeleh, H, Masoumeh Kardani, M 2016, Heavy Metals Concentrations and Human Health Risk Assessment for Three Common Species of Fish from Karkheh River, Iran, Iranian Journal of Toxicology Vol 10, No 6, November-December 2016. 